DOI: $10.217672576-3903.100005$

\title{
Optimal Colonic Cancer Surgery- the Concept of Complete Mesocolic Excision
}

\author{
Nicolae Bacalbașa ${ }^{1}$, Beatrice Lintoiu ${ }^{2}$ and Irina Balescu ${ }^{3}$ \\ ${ }^{1}$ Carol Davila University of Medicine and Pharmacy, Bucharest, Romania \\ ${ }^{2}$ Clinical Emergency Hospital, Bucharest, Romania \\ ${ }^{3}$ Ponderas Hospital, Bucharest, Romania
}

Corresponding author: Nicolae Bacalbasa, Carol Davila University of Medicine and Pharmacy, Bucharest, Romania, Tel: +40723540426; E-mail: nicolae_bacalbasa@yahoo.ro

Received date: March 22, 2016; Accepted date: April 14, 2016; Published date: April 18, 2016

Citation: Bacalbașa N, Lintoiu B, Balescu I. Optimal Colonic Cancer Surgery- the Concept of Complete Mesocolic Excision. J Neoplasm 2016, 1:1

\begin{abstract}
Although colorectal cancer is one of the most common malignancies worldwide, the colon cancer related mortality significantly decreased in the last decades due to the wide implementation of screening programs and due to a better therapeutic approach. Once Heald introduced the concept of total mesorectal excision and demonstrated its' benefits in terms of oncological outcomes, attention was focused on the possibility of implementation of a similar technique for patients with colon cancer. This is how the concept of complete mesocolic excision appeared. This is a literature review regarding the surgical technique and oncologic outcomes of complete mesocolic excision.
\end{abstract}

\section{Keywords: Colon cancer; Mesocolic excision; Survival}

\section{Introduction}

Colorectal cancer is a major health problem, being the third most frequent cancer in men $(764,000$ cases/year) and the second in women $(614,000$ cases/year) [1]. The mortality decreased with almost 30\% from 1990 until 2007 due to screening programs and to better therapeutic approaches. Over recent years, patient outcomes after colonic cancer resection have not improved to the same degree as for rectal cancer. Emil Theodor Kocher first theorized that oncologic resection is removal not only of the organ but also of its lymphatic drainage, a concept substantiated by Miles [2], Jamieson and Dobson [3] for colorectal cancers. After 70 years, a new revolution in radical surgery was performed by Heald et al. [4], who introduced the concept of total mesorectal excision (TME), the primitive embryological dorsal mesentery of the rectum, reaching significant local control, 5-year overall survival and disease-free survival [5]. The benefits in terms of long survival rates after applying the technique of TME were widely demonstrated: while in the 1980's patients with colon cancer were thought to have a significantly better outcome in terms of overall survival, once the TME has been implemented, better outcomes were reported among patients with rectal cancer $[6,7]$. Once the benefit of TME has been widely demonstrated, a new surgical technique for colon cancer has been suggested. The procedure, known under the generic name of total mesocolic excision, consists of an extended dissection of the affected colic segment carried out in the embryologically defined mesocolic planes in order to remove all the lymphatic trunks situated along the vessels which provide the blood supply of the region [4]. In this way the resected specimen is characterized by a greater distance between the tumor and the site of ligation of the main blood vessels [8]. However, initially the method remained controversial [8]. For example, in Denmark the concept of complete mesocolic excision for colon cancer was implemented in 2008 in a single center (Hillerod) while the other three centers in the Capital Region of Denmark refused the implementation of the technique. One year later, the quality of the specimens in the four centers was examined by the pathologists and significant differences in terms of resected lymph nodes and of distance between the tumor and the vascular tie being reported between the two groups [8].

\section{The concept of complete mesocolic excision}

In 2009, translating the concept of TME, came the first report and description of the complete mesocolic excision (CME) with central vascular ligation (CVL) from the Erlanger group of Hohenberger with impressive oncological outcomes and an overall 5 year survival reaching up to $70 \%$ for stage III colon cancer patients. They also demonstrated that this is a safe and feasible technique with at least the same morbidity and mortality as the "so called" standard technique. The concept of CME with CVL consists of creating an intact specimen encompassing the primary neoplasia along with all the potential pathways of tumor spread through central ligation of the main artery at its origin $[9,10]$. CME with CVL consists of two main components: the preservation of intact fasciae of the mesocolon between which relevant lymph nodes are contained in association with ligation of the blood vessels that supply the tumor colon site at their origin: at the level of the superior mesenteric vein for right sided neoplasms and at the level of their origin in the inferior mesenteric artery for left sided neoplasm [11]. Other studies advocate that there are three separate components defining the optimal CME surgery: the first one refers to the preservation of the integrity of the 
mesocolic plane during dissection, the second one refers to the central ligation of the tumor feeding blood vessel while the third refers to the removal of an appropriate length of large bowel on both sides of the tumor [12]. However, while the first component is widely accepted, the other two references have been contested by some authors: although some authors strongly sustain the benefits of central vein ligation $[13,14]$, other studies show no difference between high and low ligation $[15,16]$. As for the length of removed colon, the Japanese studies propose alternative principles regarding the amount of normal colon resected [17].

In 2012 Culligan et al. [18] published a study regarding the anatomic landmarks of the mesocolic which should be respected in patients undergoing mesocolic excision for malignant tumors. In a more recent study conducted by the same author it is demonstrated that the classic affirmation that the term of mesocolon should be applied only for the freely mobile segments of the colon is in fact a misconception, both right and left colon presenting a proper mesocolon which present in adulthood in almost all cases [19]. However, this aspect was first described by Carl Toldt in 1879 in "An Atlas of Human Anatomy for Students and Physicians" [20,21]; according to the anatomical studies, Toldt's fascia should be considered as the landmark between the apposed portions of the mesocolon and the underlying retroperitoneum. Demonstrating the mesocolic continuity and the presence of Toldt's fascia located between the apposed portions of the mesocolon and the retroperitoneum, rationalize dissection in this plan during colonic resection. The concept for CME is the consequent surgical mobilization by sharp dissection of the visceral fascia layer from the parietal one resulting in complete removal of the entire mesocolon covered by an intact visceral fascia layer, providing in this way a safe exposure and a proper ligation of the supplying arteries at their origin [18].

The extent of the surgical procedures is determined by the location of the cancer and the pattern of potential spread. For right sided tumors the procedure includes performing a Kocher maneuver and identifying the mesenteric root up to the origin of the superior mesenteric artery and exposure of superior mesenteric vein. During these surgical steps the integrity of the mesocolon should be strictly preserved [22]. Intact mesocolon in colon cancer resections is associated with a $15 \%$ better overall survival at 5 years compared with cases with mesocolic defects [23].

In the study conducted by Munkedal et al. [12] evaluating the effectiveness of the introduction of the concept of CME conducted in Aarhus University Hospital, Denmark, 209 patients with colon cancer were introduced. According to the integrity of mesocolon, the resected specimens were classified in three groups: the mesocolic plane - the specimen has an intact meseocolon with peritoneal-lined surface and no defects deeper than $5 \mathrm{~mm}$, the intramesocolic plane - the specimen has a moderate amount of mesocolon, with deeper than $5 \mathrm{~mm}$ defects, none of them reaching the muscularis propria and the muscularis propria plane - the specimen presents a small amount of mesocolon with disruptions exposing the muscularis propria. The histopathological studies revealed that an intact mesocolic plane was achieved in $61 \%$ of cases, an intramesocolic plane was achieved in $36 \%$ of cases while in the other $3 \%$ of cases the dissection was performed in the muscularis propria plane. The rate of cases in which an intact mesocolic plane was found was slightly higher for patients with right sided tumors when compared to those with left sided tumors $(p=0.075)$ [12].

In the study conducted by Bertelsen et al. [8] the authors compared the results obtained in a group of 364 patients with colic cancer submitted to CME with those obtained in a similar group of 1031 patients in whom the technique of CME was not applied. The 4 year disease free survival rate was significantly higher for patients submitted to CME among all UICC (Union for International Cancer Control) stages of the disease. Although it did not reach statistical significance, the 5 year overall survival rate was higher for patients submitted to $\mathrm{CME}$ versus cases with non-CME surgery $97.49 \%$ versus $69.8 \%$, $\mathrm{p}=0.12$ ) [8].

The surgical resection of colon cancer continues to lack international standardization. In centers undertaking meticulous mesocolic dissection with optimal lymph node clearance, outcomes are significantly better, with 5-year cancer-related survival of up to $89 \%$. Thus, CME with CVL improves the quality of the specimen and oncologic results without increasing the postoperative complication or mortality rates [17].

According to NCCN-2016, for resectable non-metastatic colon cancer, the preferred surgical procedure is colectomy with en bloc removal of the regional lymph nodes. The extent of colectomy should be based on the tumour location, resecting the portion of the bowel and arterial arcade containing the regional lymph nodes. Other nodes, such as those at the origin of the vessels feeding the tumor (ie, apical lymph node), and suspicious lymph nodes outside the field of resection, should also be biopsied or removed if possible. Resection must be complete to be considered curative, and positive lymph nodes left behind indicate an incomplete (R2) resection $[24,25]$.

\section{The extent of the lymph node dissection}

Longitudinal spread greater than $10 \mathrm{~cm}$ beyond the tumor is extremely rare, $1 \%$ to $4 \%$ for right-sided tumors and $0 \%$ for left-sided tumors [26]. The location of nodal metastases influences survival. Involved nodes situated along the major vessels have a worse prognosis than involved pericolic nodes, and this is independent of the number of nodes involved $[27,28]$. The Japanese Society for Cancer of the Colon and Rectum (JSCCR) advocates careful dissection along embryologic tissue planes, the radicality of the lymph node dissection being determinate by the clinical preoperative staging. It is recommended a D2 resection (standard low tie) for early stage tumors (stage I) and a D3resection (high tie) for more advanced disease (stage II and III) [29]. The D2-level lymph node dissection consists in the ligation of the ileocolic artery and right colic artery (if present) on the right side of the superior mesenteric vein with the removal of the epicolic, paracolic and intermediate lymph nodes. In D3 level 
dissection, the division of the ileocolic artery and right colic artery is performed to the left side of the superior mesenteric vein with additional removal of the main lymph nodes, where metastasis has been found to occur in $8 \%$ to $17 \%$ of the cases $[26,30]$. Precise evaluation of lymph nodes in the surgical specimen is crucial for the staging and subsequent decision about the adjuvant therapy in colorectal cancer.

D3 dissection was reported significantly superior to D2 dissection in terms of overall survival for patients with T3 and T4 colon cancer [31]. Excellent longterm outcome of D3 dissection in right hemicolectomy were reported by Kanemitsu and colleagues [32] from a single-institute non-randomized retrospective study in Japan. Japanese D3 surgery is based on similar principles to CME with CVL, both aiming to follow embryologic tissue planes with central ligation of the feeding arteries. Lymph node yield has been proposed as a marker for surgical quality, although it is dependent on the quality of pathologic examination and patient/tumor factors (age, microsatellite instability status, tumor size, disease stage) [33]. A higher nodal yield is consistently noted in right-sided tumors compared with left-sided tumors, partially due to a combination of greater tumor size, immune stimulation by microsatellite unstable tumors, and the anatomic presence of two vascular arcades in right-sided specimens as compared with left-sided specimen.

The establishment of CME with CVL in the mind of many, at first skeptical, colorectal surgeons came when the leading group of pathologist from Leeds [9], which is involved in almost all the techniques used by pathologist to assess and measure surgical quality in rectal surgery, published the first morphometric criteria of macroscopical and microscopical assessment of colon cancer specimens. In this way, the superiority of CME with CVL, as far as the quality of the surgical specimen is concerned, was proven.

\section{Conclusions}

Standardized oncologic surgery shows particular importance by reducing the rate of local recurrence and achieving increased survival. It is important to remember that colon cancer treatment today is multimodal and that the improvement in patients' survival in the last decades is surely linked with the improvement in chemotherapy and the advances in the agents used. However, optimal surgery is with no doubt an important element of good oncologic outcome, as the experience with rectal cancer treatment has taught us.

\section{References}

1. Ferlay J, Soerjomataram I, Dikshit R, Eser S, Mathers C, et al. (2015) Cancer incidence and mortality worldwide: sources, methods and major patterns in GLOBOCAN 2012. Int J Cancer 136: 359-386.

2. Miles WE (1971) A method of performing abdomino-perineal excision for carcinoma of the rectum and of the terminal portion of the pelvic colon. CA Cancer J Clin 21: 361-364.
3. Jamieson JK, Dobson JF (1909) Lymphatics of the Colon: With special reference to the operative treatment of cancer of the colon. Ann Surg 50: 1077-1090.

4. Heald RJ, Husband EM, Ryall RD (1982) The mesorectum in rectal cancer surgery--the clue to pelvic recurrence? Br J Surg 69: 613-616.

5. Quirke P, Steele R, Monson J, Grieve R, Khanna S, et al. (2009) Effect of the plane of surgery achieved on local recurrence in patients with operable rectal cancer: a prospective study using data from the MRC CRO7 and NCIC-CTG CO16 randomised clinical trial. Lancet 7: 821-828.

6. Heald RJ, Ryall RD (1986) Recurrence and survival after total mesorectal excision for rectal cancer. Lancet 28: 1479-1482.

7. Heald RJ (1988) The 'Holy Plane' of rectal surgery. J R Soc Med 81: 503-508.

8. Bertelsen CA, Neuenschwander AU, Jansen JE, Wilhelmsen $M$, Kirkegaard-Klitbo A, et al. (2015) Disease-free survival after complete mesocolic excision compared with conventional colon cancer surgery: a retrospective, population-based study. Lancet Oncol 16: 161-168.

9. West NP, Hohenberger W, Weber K, Perrakis A, Finan PJ, et al. (2010) Complete mesocolic excision with central vascular ligation produces an oncologically superior specimen compared with standard surgery for carcinoma of the colon. J Clin Oncol 28: 272-278.

10. Morikawa E, Yasutomi M, Shindou K, Matsuda T, Mori N, et al. (1994) Distribution of metastatic lymph nodes in colorectal cancer by the modified clearing method. Dis Colon Rectum 37: 219-223.

11. Hohenberger W, Weber K, Matzel K, Papadopoulos T, Merkel S, et al. (2009) Standardized surgery for colonic cancer: complete mesocolic excision and central ligation--technical notes and outcome. Colorectal Dis 11: 354-364.

12. Munkedal DL, West NP, Iversen LH, Hagemann-Madsen R, Quirke P, Laurberg S. Implementation of complete mesocolic excision at a university hospital in Denmark: An audit of consecutive, prospectively collected colon cancer specimens. Eur J Surg Oncol 40: 1494-1501.

13. Kanemitsu Y, Komori K, Kimura K, Kato T (2013) D3 Lymph Node Dissection in Right Hemicolectomy with a No-touch Isolation Technique in Patients With Colon Cancer. Dis Colon Rectum 56: 815-824.

14. Slanetz CA, Jr.,Grimson R (1997) Effect of high and intermediate ligation on survival and recurrence rates following curative resection of colorectal cancer. Dis Colon Rectum 40: 1205-1218.

15. Pezim ME, Nicholls RJ (1984) Survival after high or low ligation of the inferior mesenteric artery during curative surgery for rectal cancer. Ann Surg 6: 729-733.

16. Surtees P, Ritchie JK, Phillips RK (1990) High versus low ligation of the inferior mesenteric artery in rectal cancer. Br J Surg 77: 618-621.

17. West NP, Kobayashi H, Takahashi K, Perrakis A, Weber K, et al. (2012) Understanding optimal colonic cancer surgery: comparison of Japanese D3 resection and European complete mesocolic excision with central vascular ligation. J Clin Oncol 30: 1763-1769.

18. Culligan K, Coffey JC, Kiran RP, Kalady M, Lavery IC, et al (2012) The mesocolon: a prospective observational study. Colorectal Dis 14: 421-428. 
19. Culligan K, Remzi FH, Soop M, Coffey JC (2013) Review of nomenclature in colonic surgery-proposal of a standardised nomenclature based on mesocolic anatomy. Surgeon 11: 1-5.

20. Toldt C (1879) Bound growth vera expansions of the human gekrose metallic intestinal canal. Denks 41: 156.

21. Toldt C (1919) An atlas of human anatomy for students and physicians. 4: 408.

22. West NP, Hohenberger W, Finan PJ, Quirke P (2009) Mesocolic plane surgery: an old but forgotten technique? Colorectal Dis 11: 988-989.

23. West NP, Morris EJ, Rotimi O, Cairns A, Finan PJ, et al. (2008) Pathology grading of colon cancer surgical resection and its association with survival: a retrospective observational study. Lancet Oncol 9: 857-865.

24. Edge SB, Compton CC (2010) The American Joint Committee on Cancer: The AJCC cancer staging manual and the future of TNM. (7thedn) Ann Surg Oncol 17: 1471-1474.

25. Berger AC, Sigurdson ER, LeVoyer T, Hanlon A, Mayer RJ, et al. (2005) Colon cancer survival is associated with decreasing ratio of metastatic to examined lymph nodes. J Clin Oncol 23: 8706-8712.

26. Toyota S, Ohta H, Anazawa S (1995) Rationale for extent of lymph node dissection for right colon cancer. Dis Colon Rectum 38: 705-711.
27. Kobayashi H, Ueno H, Hashiguchi $Y$, Mochizuki H (2006) Distribution of lymph node metastasis is a prognostic index in patients with stage III colon cancer. Surgery 139: 516-522.

28. Kobayashi $H$, Enomoto $M$, Higuchi $T$, Uetake $H$, lida $S$, et al. (2011) Clinical significance of lymph node ratio and location of nodal involvement in patients with right colon cancer. Dig Surg 28: 190-197.

29. JSCCR (2009) Japanese Society for Cancer of the Colon and Rectum: Japanese classification of colorectal carcinoma. Kanehara, Tokyo, Japan.

30. Rose J, Schneider C, Yildirim C, Geers P, Scheidbach H, et al. (2004) Complications in laparoscopic colorectal surgery: results of a multicentre trial. Tech Coloproctol 8 1: 25-28.

31. Kotake K, Mizuguchi T, Moritani K, Wada O, Ozawa $\mathrm{H}$, et al. (2014) Impact of D3 lymph node dissection on survival for patients with T3 and T4 colon cancer. Int J Colorectal Dis 29: 847-852.

32. Kanemitsu Y, Hirai T, Komori K, Kato T (2006) Survival benefit of high ligation of the inferior mesenteric artery in sigmoid colon or rectal cancer surgery. Br J Surg 93: 609-615.

33. West $N$ (2010) Lymph node retrieval in colorectal cancer specimens: national standards are achievable, and low numbers are associated with reduced survival. Colorectal Dis 12: 309-311. 